\title{
SOME PROPERTIES ON THE TENSOR SQUARE OF LIE ALGEBRAS
}

\author{
PEYMAN NIROOMAND
}

\begin{abstract}
In the present paper we extend and improve the results of [2, 4] for the tensor square of Lie algebras. More precisely, for any Lie algebra $L$ with $L / L^{2}$ of finite dimension, we prove $L \otimes L \cong L \square L \oplus L \wedge L$ and $Z^{\wedge}(L) \cap L^{2}=$ $Z^{\otimes}(L)$. Moreover, we show that $L \wedge L$ is isomorphic to derived subalgebra of a cover of $L$, and finally we give a free presentation for it.
\end{abstract}

\section{INTRODUCTION AND PRELIMINARIES}

Let $L$ and $H$ be Lie algebras over a fixed field $F$ and let [,] denotes the Lie bracket. A bilinear map $\rho: L \times L \rightarrow H$ is said to be a Lie pairing provided that

(i) $\rho\left(\left[l, l^{\prime}\right], s\right)=\rho\left(l,\left[l^{\prime}, s\right]\right)-\rho\left(l^{\prime},[l, s]\right)$,

(ii) $\rho\left(l,\left[s, s^{\prime}\right]\right)=\rho\left(\left[s^{\prime}, l\right], s\right)-\rho\left([s, l], s^{\prime}\right)$,

(iii) $\rho\left([l, s],\left[l^{\prime}, s^{\prime}\right]\right)=-\left[\rho(s, l), \rho\left(l^{\prime}, s^{\prime}\right)\right]$, for all $l, l^{\prime}, s, s^{\prime} \in L$.

For instance it can be checked that the map $L \times L \rightarrow L^{2}$ is a Lie pairing.

The theory of tensor square of groups was extended to tensor square of Lie algebras by Ellis in [6].

Specifically, the tensor square $L \otimes L$ is a Lie algebra generated by the symbols $l \otimes k$ subject to the following relations

(i) $c(l \otimes k)=c l \otimes k=l \otimes c k$,

(ii) $\left(l+l^{\prime}\right) \otimes k=l \otimes k+l^{\prime} \otimes k$, $l \otimes\left(k+k^{\prime}\right)=l \otimes k+l \otimes k^{\prime}$

(iii) $\left[l, l^{\prime}\right] \otimes k=l \otimes\left[l^{\prime}, k\right]-l^{\prime} \otimes[l, k]$, $l \otimes\left[k, k^{\prime}\right]=\left(\left[k^{\prime}, l\right]\right) \otimes k-[k, l] \otimes k^{\prime}$,

(iv) $\left[(l \otimes k),\left(l^{\prime} \otimes k^{\prime}\right)\right]=-[k, l] \otimes\left[k^{\prime}, l^{\prime}\right]$ for all $c \in F, l, l^{\prime}, k, k^{\prime} \in L$.

Recall from [6, Proposition 1] the mapping

$$
h: L \times L \rightarrow L \otimes L,\left(l, l^{\prime}\right) \mapsto l \otimes l^{\prime}
$$

Date: October 30, 2018.

Key words and phrases. Tensor square of Lie algebra, Schur multiplier of Lie algebra. Mathematics Subject Classification 2010. Primary 17B60; 17 B99. 
is a universal Lie pairing, that is for any Lie pairing $h^{\prime}: L \times L \rightarrow Q$ there is a unique Lie homomorphism $\zeta: L \otimes L \rightarrow Q$ such that $\zeta h=h^{\prime}$.

Let $L \square L$ denotes the submodule of $L \otimes L$ generated by $l \otimes l$ for all $l \in L$. Then the exterior square $L \wedge L$ of $L$ is the quotient $L \otimes L / L \square L$, for $l \otimes l^{\prime} \in L \otimes L$ we denote the coset $l \otimes l^{\prime}+L \square L$ by $l \wedge l^{\prime}$.

Ellis in [6] defined $J_{2}(L)=\operatorname{ker}\left(L \otimes L \stackrel{\kappa}{\rightarrow} L^{2}, l \otimes l^{\prime} \mapsto\left[l, l^{\prime}\right]\right)$. The kernel of $\kappa$ contains $L \square L$, thus $\kappa$ induces an epimorphism $\kappa^{\prime}: L \wedge L \rightarrow L^{2}$. It is known that the Schur multiplier $\mathcal{M}(L)$ is the kernel of $\kappa^{\prime}$ by the result of 5 .

The concept of Whitehead's quadratic functor $\Gamma$ (as defined in 14] for groups and [6] for Lie algebras) gives the natural epimorphism $\Gamma\left(L / L^{2}\right) \rightarrow L \square L$ defined by $\gamma\left(L^{2}+l\right) \mapsto l \otimes l$ and helps to survey the connection between $L \square L$ and $L / L^{2} \square L / L^{2}$.

Several authors tried to extend known results of groups to Lie algebras (see for instance [1, 3, 5, 6, 7, 8, 9, 10, 11, 12, 13).

The present paper is devoted to develop and improve an analogous theory of tensor products from [2, 4] for Lie algebras. More precisely, we obtain strong Lie algebra version of 2 that is

$$
L \otimes L \cong L \square L \oplus L \wedge L \text { and } Z^{\wedge}(L) \cap L^{2}=Z^{\otimes}(L)
$$

where $L / L^{2}$ is a finite dimensional Lie algebra and the tensor and exterior centers of a Lie algebra $L$ are defined as follows

$$
\begin{aligned}
& Z^{\otimes}(L)=\left\{l \in L \mid l \otimes l^{\prime}=0, \text { for all } l^{\prime} \in L\right\} \text { and } \\
& Z^{\wedge}(L)=\left\{l \in L \mid l \wedge l^{\prime}=0, \text { for all } l^{\prime} \in L\right\},
\end{aligned}
$$

respectively. Moreover, we prove that $L \wedge L$ is isomorphic to derived subalgebra of a cover of $L$, and we give a free presentation for the exterior square analogues to [4, 2].

The notation and terminology which are used throughout this paper is standard and follows [5, 6, 9 .

\section{Main Results}

This section is devoted to develop and improve a Lie algebra version of [2, 4.

First we state the following two lemmas which will be used in the rest.

Lemma 2.1. (See 9, Corollary 3.2]) Let L be a finite dimensional Lie algebra and $\pi: L \otimes L \rightarrow L / L^{2} \otimes L / L^{2}$ be the natural epimorphism. Then the restriction $\pi: L \square L \rightarrow L / L^{2} \square L / L^{2}$ is an isomorphism.

Lemma 2.1 can be improved as follows

Lemma 2.2. Let $L$ be a Lie algebra such that $L / L^{2}$ is of finite dimension. Then the restriction $\pi: L \square L \rightarrow L / L^{2} \square L / L^{2}$ is an isomorphism. 
Proof. The natural epimorphism $\Gamma\left(L / L^{2}\right) \rightarrow L \square L$ guarantees $L \square L$ to be of finite dimension, and hence the restriction $\pi: L \square L \rightarrow L / L^{2} \square L / L^{2}$ is an isomorphism by Lemma 2.1

Lemma 2.3. Let $L$ be an abelian Lie algebra with a basis $\left\{x_{1}, x_{2}, \ldots, x_{n}\right\}$. Then

$$
L \otimes L \cong L \square L \oplus\left\langle x_{i} \otimes x_{j} \mid 1 \leq i<j \leq n\right\rangle .
$$

Proof. Since $L \cong \bigoplus_{i=1}^{n}\left\langle x_{i}\right\rangle$, we have

$$
L \otimes L \cong\left\langle x_{i} \otimes x_{j}+x_{j} \otimes x_{i}, x_{i} \otimes x_{i} \mid 1 \leq i<j \leq n\right\rangle \oplus\left\langle x_{i} \otimes x_{j} \mid 1 \leq i<j \leq n\right\rangle .
$$

The result follows from the fact $L \square L \cong\left\langle x_{i} \otimes x_{j}+x_{j} \otimes x_{i}, x_{i} \otimes x_{i} \mid 1 \leq i<j \leq n\right\rangle$.

Lemma 2.4. Let $L$ be a Lie algebra and let $\pi: L \otimes L \rightarrow L / L^{2} \otimes L / L^{2}$ be the natural epimorphism. Then $\operatorname{Ker} \pi=L \otimes L^{2}+L^{2} \otimes L=L \otimes L^{2}$.

Proof. Taking $N=L \otimes L^{2}+L^{2} \otimes L$. The epimorphism $\pi$ has $N$ in its kernel and $N$ is an ideal of $L \otimes L$ by invoking (iv), therefore inducing a map

$$
\bar{\pi}:(L \otimes L) / N \rightarrow L / L^{2} \otimes L / L^{2} .
$$

On the other hand, let $\bar{x}$ denotes $x$ modulo $L^{2}$. Define

$$
\alpha: L / L^{2} \times L / L^{2} \rightarrow(L \otimes L) / N \text { by }(\bar{x}, \bar{y}) \mapsto N+x \otimes y .
$$

It readily shown that $\alpha$ is well-defined. Let $l, l^{\prime}, k, k^{\prime} \in L$, then

$$
\begin{aligned}
\alpha\left(\left[\bar{l}, \bar{l}^{\prime}\right], k\right) & =N+\left[l, l^{\prime}\right] \otimes k=N+\left(l \otimes\left[l^{\prime}, k\right]-l^{\prime} \otimes[l, k]\right) \\
& =N+l \otimes\left[l^{\prime}, k\right]-N+l^{\prime} \otimes[l, k]=\alpha\left(\bar{l},\left[\overline{l^{\prime}}, \bar{k}\right]\right)-\alpha\left(\overline{l^{\prime}},[\bar{l}, \bar{k}]\right) .
\end{aligned}
$$

By a similar fashion, it is easily seen that

$$
\alpha\left(\bar{l},\left[\bar{k}, \overline{k^{\prime}}\right]\right)=\alpha\left(\left[\bar{k}^{\prime}, l\right], k\right)-h\left([\bar{k}, \bar{l}], \bar{k}^{\prime}\right) \text { and } \alpha\left([\bar{k}, \bar{l}],\left[\bar{l}^{\prime}, \bar{k}^{\prime}\right]\right)=-\left[\alpha(l, k), \alpha\left(\bar{l}^{\prime}, \bar{k}^{\prime}\right)\right] .
$$

Thus $\alpha$ is a Lie pairing, and hence induces a Lie homomorphism $\bar{\alpha}: L / L^{2} \otimes L / L^{2} \rightarrow$ $(L \otimes L) / N$ by using [13, Lemma 1.1]. Hence $(L \otimes L) / N \cong L / L^{2} \otimes L / L^{2}$ since $\bar{\pi} \bar{\alpha}$ and $\bar{\alpha} \bar{\pi}$ are identity. The equality $L \otimes L^{2}+L^{2} \otimes L=L \otimes L^{2}$ is obtained directly from relations of $L \otimes L$

Theorem 2.5. Let $L / L^{2}$ be a finite dimensional Lie algebra. Then

$$
L \otimes L \cong L \wedge L \oplus L \square L .
$$

Proof. By invoking Lemma 2.3, we have

$$
L / L^{2} \otimes L / L^{2} \cong L / L^{2} \square L / L^{2} \oplus\left\langle\overline{x_{i}} \otimes \overline{x_{j}} \mid 1 \leq i<j \leq n\right\rangle .
$$


Now consider the map $\pi: L \otimes L \rightarrow L / L^{2} \otimes L / L^{2}$, we should have

$$
L / L^{2} \square L / L^{2} \oplus\left\langle\overline{x_{i}} \otimes \bar{x}_{j} \mid 1 \leq i<j \leq n\right\rangle=\pi\left(L \square L+\left\langle x_{i} \otimes x_{j} \mid 1 \leq i<j \leq n\right\rangle\right),
$$

where $\left\{\bar{x}_{1}, \bar{x}_{2}, \ldots, \bar{x}_{n}\right\}$ is a basis for $L / L^{2}$, and hence $L \otimes L=L \square L+\left\langle x_{i} \otimes x_{j}\right| 1 \leq$ $i<j \leq n\rangle)+N$ due to Lemma 2.4. By virtue of Lemma 2.2, the restriction of $\pi$ maps $x$ to zero for all $\left.x \in L \square L \cap\left(\left\langle x_{i} \otimes x_{j} \mid 1 \leq i<j \leq n\right\rangle\right)+N\right)$, which implies that

$$
\left.L \otimes L \cong L \square L \oplus\left(\left\langle x_{i} \otimes x_{j} \mid 1 \leq i<j \leq n\right\rangle\right)+N\right)
$$

via Lemma 2.2. It is clear that $L \wedge L \cong\left\langle x_{i} \otimes x_{j} \mid 1 \leq i<j \leq n\right\rangle+N$, as required.

Corollary 2.6. Let $L / L^{2}$ be a finite dimensional Lie algebra. Then

$$
J_{2}(L) \cong L \square L \oplus \mathcal{M}(L) .
$$

Proof. By Theorem 2.5.

$$
\begin{aligned}
J_{2}(L) & =\left(L \square L \oplus\left(\left\langle x_{i} \otimes x_{j} \mid 1 \leq i<j \leq n\right\rangle+N\right)\right) \cap J_{2}(L) \\
& =L \square L \oplus\left(\left(\left\langle x_{i} \otimes x_{j} \mid 1 \leq i<j \leq n\right\rangle+N\right) \cap J_{2}(L)\right) .
\end{aligned}
$$

The rest of proof is obtained by the fact $J_{2}(L) / L \square L \cong \mathcal{M}(L)$.

Corollary 2.7. Let $L / L^{2}$ be a finite dimensional Lie algebra. Then $Z^{\wedge}(L) \cap L^{2}=$ $Z^{\otimes}(L)$

Proof. Let $l \in Z^{\wedge}(L) \cap L^{2}$. Then for all $l^{\prime} \in L$ we have $l \otimes l^{\prime} \in L \square L$, and also $l \otimes l^{\prime} \in \operatorname{Ker} \pi$. Thus Lemma 2.2 implies that $l \otimes l^{\prime}=0$, and hence $l \in Z^{\otimes}(L)$. Conversely $Z^{\otimes}(L) \subseteq L^{2}$ since for all $l \in Z^{\otimes}(L)$, we have $l+L^{2} \otimes l^{\prime}+L^{2}=0$ which implies that $l \in L^{2}$. The result follows.

A pair of Lie algebras $(K, M)$ is called a defining pair for $L$ if

(i) $0 \rightarrow M \rightarrow K \rightarrow L \rightarrow 0$ is exact;

(ii) $M \subseteq Z(K) \cap K^{2}$.

When $L$ is finite dimensional then the dimension of $K$ is bounded. The $K$ of maximal dimension is called a cover of $L$, and the corresponding $M$, is the Schur multiplier of L. From [1, the Schur multiplier of $L$ can be also defined in term of free Lie algebra. More precisely, for an exact sequence

$$
0 \rightarrow R \rightarrow F \rightarrow L \rightarrow 0
$$

where $F$ is a free Lie algebra, the Schur multiplier of $L$ is isomorphic to the factor Lie algebra $R \cap F^{2} /[R, F]$. It is known by [7] that for a finite dimension Lie algebra Lie covers always exist and are unique up to isomorphism. 
We are interested in proving that the derived subalgebra of a covering Lie algebra of $L$ is isomorphic to $L \wedge L$. These developes the results which have been obtained in 2 , 4. First we recall the following proposition from 5 .

Proposition 2.8. Let $F$ be a free Lie algebra. Then

$$
F^{2} \rightarrow F \wedge F,[x, y] \mapsto x \wedge y
$$

is an isomorphism.

Theorem 2.9. Let $L$ be a Lie algebra with a covering Lie algebra $\bar{L}$. Then

$$
L \wedge L \cong \bar{L}^{2} .
$$

Proof. Let $\bar{L}$ be a cover of $L$. Then there is an exact sequence

$$
0 \rightarrow \mathcal{M}(L) \rightarrow \bar{L} \rightarrow L \rightarrow 0
$$

Suppose that $\bar{L} \cong F / R$ where $F$ is a free Lie algebra, so $L$ is isomorphic to $F / S$ in which $\mathcal{M}(L) \cong S / R$.

By invoking Proposition 2.8, there is a Lie homomorphism

$$
\eta: F^{2} \rightarrow F / S \wedge F / S
$$

sending $\alpha_{1}\left[x_{1}, y_{1}\right]+\ldots+\alpha_{k}\left[x_{k}, y_{k}\right]$ to $\alpha_{1}\left(S+x_{1} \wedge S+y_{1}\right)+\ldots+\alpha_{k}\left(S+x_{k} \wedge S+y_{k}\right)$. Evidently, $\eta$ factors through $R \cap F^{2}$, to induces a homomorphism

$$
\bar{\eta}: \frac{R \cap F^{2}}{R} \rightarrow F / S \wedge F / S .
$$

From the definition of $\bar{\eta}$, its surjectivity follows immediately. We claim that $\bar{\eta}$ is injective.

Define $\theta: F / S \times F / S \rightarrow \frac{F^{2}}{R \cap F^{2}}$ by $\theta\left(S+f, S+f_{1}\right)=R \cap F^{2}+\left[f, f_{1}\right]$. Since $[S, F] \subseteq R \cap F^{2}$, one can easily check that $\theta$ is well-defined. Since $h: F / S \times$ $F / S \rightarrow F / S \otimes F / S$ is a universal Lie pairing, there exists a Lie homomorphism $\tau: F / S \otimes F / S \rightarrow \frac{F^{2}}{R \cap F^{2}}$ such that $\tau h=\theta$. Of course, $\tau$ is trivial on the ideal $F / S \square F / S$, and so it induces a Lie homomorphism

$$
\bar{\tau}: F / S \wedge F / S \rightarrow \frac{F^{2}}{R \cap F^{2}} .
$$

Now both of $\bar{\tau} \bar{\eta}$ and $\bar{\eta} \bar{\tau}$ are identity, and it implies that

$$
F / S \wedge F / S \cong \frac{F^{2}}{R \cap F^{2}} \cong \frac{F^{2}+R}{R},
$$

as required. 
Theorem 2.10. Let $0 \rightarrow R \rightarrow F \rightarrow L \rightarrow 0$ be a free presentation of a Lie algebra L. Then

$$
L \wedge L \cong F^{2} /[R, F]
$$

Proof. Using Proposition 2.8, there is a Lie homomorphism $\varepsilon: F^{2} \rightarrow F / R \wedge F / R$ sending $\alpha_{1}\left[x_{1}, y_{1}\right]+\ldots+\alpha_{k}\left[x_{k}, y_{k}\right]$ to $\alpha_{1}\left(R+x_{1} \wedge R+y_{1}\right)+\ldots+\alpha_{k}\left(R+x_{k} \wedge R+y_{k}\right)$. Since $\varepsilon$ sends $[R, F]$ to identity, it induces a Lie homomorphism $\bar{\varepsilon}: F^{2} /[R, F] \rightarrow$ $F / R \wedge F / R$. On the other hand, since $\xi: F / R \times F / R \rightarrow F^{2} /[R, F]$ is a Lie pairing,

obviously there exists a Lie homomorphism $\bar{\xi}: F / R \wedge F / R \rightarrow F^{2} /[R, F]$ sending $R+f \wedge R+f_{1}$ to $[R, F]\left[f, f_{1}\right]$. It is readily obtained that $\bar{\varepsilon} \bar{\xi}$ and $\bar{\xi} \bar{\varepsilon}$ are identity, and the proof is complete.

\section{REFERENCES}

[1] P. Batten, Multipliers and covers of Lie algebras, dissertation, North Carolina State University (1993).

[2] R.D. Blyth, F. Fumagalli and M. Morigi, Some structural results on the non-abelian tensor square of groups. J. Group Theory 13(1) (2010), 83-94.

[3] L.R. Bosko, On Schur multipliers of Lie algebras and groups of maximal class. Internat. J. Algebra Comput. 20 (2010) 807-821.

[4] R. Brown, D. L. Johnson and E. F. Robertson, Some computations of nonabelian- tensor products of groups. J. Algebra 111 (1987), 177-202.

[5] G. Ellis, Non-abelian exterior products of Lie algebras and an exact sequence in the homology of Lie algebras. J. Pure Appl. Algebra 46 (1987), 111-115.

[6] G. Ellis, A non-abelian tensor product of Lie algebras. Glasgow Math. J. 33 (1991), 101-120.

[7] K. Moneyhun, Isoclinisms in Lie algebras. Algebras Groups Geom. 11 (1994), no. 1, 922.

[8] P. Niroomand, On dimension of the Schur multiplier of nilpotent Lie Algebras. Cent. Eur. J. Math. 91 (2011) 57-64.

[9] P. Niroomand, On the tensor square of non-abelian nilpotent finite dimensional Lie algebras. Linear Multilinear Algebra, DOI: 10.1080/03081087.2010.497491, in press.

[10] P. Niroomand, F. Russo, A note on the Schur multiplier of a nilpotent Lie algebra. Comm. Algebra 39 (2011), 1293-1297

[11] P. Niroomand, F. Russo, A restriction on the Schur multiplier of nilpotent Lie algebra. Elecrton. J. Linear Algebra, 22 (2011), 1-9

[12] A.R. Salemkar, H. Bigdely and V. Alamian, Some properties on isoclinism of Lie algebras and covers. J. Algebra Appl. 7 (2008) 507-516

[13] A.R. Salemkar, H. Tavallaee, H. Mohammadzadeh and B. Edalatzadeh, On the non-abelian tensor product of Lie algebras, Linear Multilinear Algebra 58 (2010) 333-341.

[14] J.H.C. Whitehead, A certain exact sequence., Ann. of Math. 52 (1950), 51-110.

School of Mathematics and Computer Science, Damghan University, Damghan, Iran E-mail address: p_niroomand@yahoo.com, niroomand@du.ac.ir 